\title{
Sentiment Classification of News Text Data Using Intelligent Model
}

\author{
Shitao Zhang* \\ School of Network Communication, Zhejiang Yuexiu University, Shaoxing, China
}

Text sentiment classification is a fundamental sub-area in natural language processing. The sentiment classification algorithm is highly domain-dependent. For example, the phrase "traffic jam" expresses negative sentiment in the sentence "I was stuck in a traffic jam on the elevated for 2 h." But in the domain of transportation, the phrase "traffic jam" in the sentence "Bread and water are essential terms in traffic jams" is without any sentiment. The most common method is to use the domain-specific data samples to classify the text in this domain. However, text sentiment analysis based on machine learning relies on sufficient labeled training data. Aiming at the problem of sentiment classification of news text data with insufficient label news data and the domain adaptation of text sentiment classifiers, an intelligent model, i.e., transfer learning discriminative dictionary learning algorithm (TLDDL) is proposed for cross-domain text sentiment classification. Based on the framework of dictionary learning, the samples

Edited by:

Yizhang Jiang

Jiangnan University, China

Reviewed by:

Jing Xue,

Wuxi People's Hospital Affiliated to Nanjing Medical University, China

Tongguang Ni,

Changzhou University, China

*Correspondence:

Shitao Zhang

wisepro1982@163.com

Specialty section:

This article was submitted to

Emotion Science,

a section of the journal

Frontiers in Psychology

Received: 15 August 2021 Accepted: 08 September 2021 Published: 28 September 2021

Citation:

Zhang S (2021) Sentiment Classification of News Text Data Using Intelligent Model.

Front. Psychol. 12:758967. doi: 10.3389/fpsyg.2021.758967 from the different domains are projected into a subspace, and a domain-invariant dictionary is built to connect two different domains. To improve the discriminative performance of the proposed algorithm, the discrimination information preserved term and principal component analysis (PCA) term are combined into the objective function. The experiments are performed on three public text datasets. The experimental results show that the proposed algorithm improves the sentiment classification performance of texts in the target domain.

Keywords: transfer learning, text sentiment, cross-domain, intelligent model, sentiment classification

\section{INTRODUCTION}

News media platforms and various social media platforms produce a large amount of content every day, including a large number of comments generated by network users. These views and opinions often potentially express their feelings or sentiments. How to exploit users's sentimental information and analyze their sentimental tendency from these massive comment data has potential application value in many aspects. For example, government departments analyze users' attitudes toward specific events and topics, grasp public opinions, and keep abreast of public opinion to make appropriate decisions. Commercial enterprises can keep abreast of the market's reputation by evaluating the relevant content of goods and services, to further improve the quality of their products and services.

Sentiment analysis, also known as sentiment classification, is the field of study that analyses the opinions, views, and sentimental attitudes that people display in texts (Wen et al., 2020; 
Birjali et al., 2021). Conventional sentiment classification methods are roughly grouped into sentiment dictionary methods and machine learning methods (Zhang et al., 2018; Ahuja et al., 2019). The classification method based on sentiment dictionary mainly uses a manually collated and constructed sentiment dictionary library. The sentiment score of the text is then calculated according to the predetermined rules. Finally, the results of sentiment classification can be obtained based on the obtained sentiment score. There are several obvious shortcomings in the classification method based on sentiment dictionaries. The accuracy of classification results depends on the size of the whole sentiment dictionary resources. When the number of dictionary resources is not large enough, the accuracy of classification results is often not high. In addition, such methods are also difficult to deal with the implied sentimental content. The core of the machine learning methods is the effective feature extraction and classifier. By constructing the feature set, the classifier is trained on the feature set of the training set, and the sentiment label is output for the unlabeled text. In recent years, the commonly used algorithms for text sentiment analysis and text classification include deep learning (Chen et al., 2020; Zhang et al., 2020), decision tree (Almunirawi and Maghari, 2016), support vector machine (Ahmad et al., 2017), sparse representation (Unnikrishnan et al., 2019; Gu et al., 2020), KNN classifier (Bozkurt et al., 2019), Naïve Bayes (Alshamsi et al., 2020), fuzzy logic (Chaturvedi et al., 2019), and extreme learning machine (Lauren et al., 2018; Waheeb et al., 2020).

However, machine learning methods depend on a large number of high-quality label texts, and the manually labeled samples obviously cannot fully meet the needs of sentiment analysis. The expression of sentiment in a text is closely related to the described semantic concept (domain), and the description of sentiment in different domains differs significantly. The distribution of data characteristics in different fields is also different. In this case, directly using the classifiers trained in other domains for text sentiment analysis will lead to the poor adaptability problem. For example, the word "unpredictable" expresses the positive sentiment in literary and artworks, such as "This movie is both exciting and unpredictable! The film is worth seeing!" but in the field of electronic, the word "unpredictable" expresses the negative sentiment, such as "Even if the power is maintained, it is unpredictable. It's terrible!" As another example, the words "excellent" and "horrible" appear in both domains of electronic and literary works, but "high resolution" and "run fast" rarely appear in the field of literary works, and "printed well" rarely appears in the field of electronic products. Therefore, it is inappropriate to directly use the sentiment classifier trained in the electronic domain to predict the sentiment of comments in the literary works domain.

Recently, the research on cross-domain sentiment classifiers using transfer learning technology becomes a research hotspot (Meng et al., 2019). Transfer learning is a machine learning technology that extracts knowledge from the source domain (the similar but different domain) and applies it to the target domain (the current domain) (Jiang et al., 2020, 2021). The common transfer learning algorithms include sample-based algorithms and feature space-based algorithms (Fu and Liu, 2021; Zhao et al., 2021). The former includes feature selection and feature projection. The sample-based algorithm mainly selects the samples that are valuable for the classification of the target domain from the source domain. To reduce the impact of negative transfer, Gui et al. (2015) developed a negative transfer detection algorithm. This algorithm detected multiple quality levels and retained the high-quality samples. Chen et al. (2010) developed a combined feature level and instance level transfer learning algorithm. This algorithm combined the samples from different domains by evaluating the classification performance in the target domain. Tian et al. (2019) developed an instance-based algorithm for imbalanced cross-linguistic viewpoint analysis. This algorithm translated other relevant markup datasets into the target domain as the supplementary training data.

Feature space-based transfer learning methods include feature selection and feature projection (Xia et al., 2013). The purpose of feature selection is to find the shared features between different domains through some strategies; and then use these features for knowledge transfer. The feature projection strategy maps the features of each domain into a shared feature representation space and establishes the association between the features of each domain. The difference between feature projection and feature selection is that the projected features are not among the original features, but new features. Wu and Tan (2011) developed a twostage transfer learning sentiment classification algorithm. In the "bridge-building" stage, a bridge between different domains was built to obtain some of the most reliable labeled texts in the target domain. In the "structure following" stage, the internal structure was adopted to label the texts by using the obtained reliable texts. Du et al. (2020) developed a Wasserstein-based transfer network. This algorithm also used a recurrent neural network to capture useful features. López et al. (2019) developed an evolutionary ensemble algorithm for text sentiment analysis. This algorithm was built on a set of evolutionary algorithms. The optimization of the model was achieved by optimizing each sub-algorithm. Wang et al. (2018) developed a measure index called sentiment-related index to measure associations between different domains, and then used this index as a bridge between different domains of features. Tang et al. (2021) developed a graph domain adversarial transfer network for text sentiment classification. This algorithm used a gradient reversal layer to obtain the domain-invariant text features and adopted a projection mechanism to obtain the domain-independent feature representations. Fei et al. (2020) developed a deep learning structure-based transfer learning algorithm, which combined the cross-entropy and weighted for word into the recurrent neural network framework.

Dictionary learning plays a key role in sparse representation and low-rank modeling. The basic operation in sparse representation is called "sparse coding," which involves reconstructing the data representation using a sparse set of constructive blocks (called "atoms"), and the atoms are clustered in a structure called a "dictionary" (Gu et al., 2021; Ni et al., 2021). Therefore, the dictionary learning algorithm has data self-adaptability, which aims to learn a series of basic atoms 
from the dataset to linearly approximate the given data. Since dictionary learning learns a set of atoms to obtain discriminative feature representation, it performs well in classification tasks. In this study, I develop a transfer learning discriminative dictionary learning (TLDDL) algorithm for cross-domain text sentiment classification. Considering the distribution difference between different domains, I adopt the subspace technology to project all samples into a subspace. In the common subspace, a domain-invariant dictionary is built to connect different domains. Moreover, the discrimination information preserved term and PCA term are embedded into the objective function, so that the performance of TLDDL for classification tasks across domains is enhanced. TLDDL learns all parameters in an alternating iteration manner. By comparing several non-transfer learning and transfer learning algorithms on toutiao-text (Zhang et al., 2021), Chinese Weibo (Bai et al., 2020), and Amazon review (Pan et al., 2010) datasets, one can draw that the TLDDL algorithm is efficient.

The rest of this paper is organized as follows: the next section presents dictionary learning. The proposed TLDDL algorithm and its optimization are described in section "Transfer Learning Discriminative Dictionary Learning Algorithm”. Experimental results are given in section "Experiment." Finally, section "Conclusion" concludes the paper.

\section{BACKGROUNDS}

Given a data matrix $\mathbf{Y}$, the dictionary learning algorithm attempts to learn the dictionary $\mathbf{D}$ so that the data $\mathbf{Y}$ can be approximately linearly expressed as $\mathbf{Y} \approx \mathbf{D S}$. Dictionary learning can be represented as,

$$
\begin{aligned}
& \arg \min _{D, S}\|\mathbf{Y}-\mathbf{D S}\|_{F}^{2}, \\
& \text { s.t. } \forall i,\left\|s_{i}\right\|_{0} \leq T_{i},
\end{aligned}
$$

where $T_{i}$ is the given threshold. $\mathbf{S}$ is the sparse coding matrix.

The constraints are used to constrain the complexity of the dictionary matrix. Dictionary learning can also be represented as the following optimization problem, where the constraints are replaced as well as the objective function,

$$
\begin{aligned}
& \min _{D, S}\left\|\mathbf{s}_{i}\right\|_{0}, i=1, \ldots, n \\
& \text { s.t. }\|\mathbf{Y}-\mathbf{D S}\|_{F}^{2}<\varepsilon, \\
& \quad\left\|d_{j}\right\|_{2}^{2}<1, j=1, \ldots, K
\end{aligned}
$$

Where $\|\cdot\|_{0}$ represents the 0 norm of the vector. $n$ and $K$ are the number of training samples and atoms, respectively. It is difficult to optimize the 0 norm in Equations 1, 2, so the 1 norm is commonly used in dictionary learning. For the above optimization problem, after the transformation of the Lagrange equation, Equation 1 can be represented as follows,

$$
\begin{aligned}
& \min _{D, S}\|\mathbf{Y}-\mathbf{D S}\|_{F}^{2}+\lambda\left\|s_{i}\right\|_{1}, i=1, \ldots, n \\
& \text { s.t. }\left\|d_{j}\right\|_{2}^{2}<1, j=1, \ldots, K
\end{aligned}
$$

It is easily can be seen that dictionary learning minimizes the reconstruction error $\| \mathbf{Y}$-DS $\|_{F}^{2}$, while the 1 norm of sparse coding $\mathbf{s}_{i}$ is as small as possible, that is, the sparsity of $\mathbf{S}$ is as strong as possible. $\lambda$ is the trade-off parameter. The optimization process of dictionary learning generally includes two alternating iterative steps, one is to solve sparse coding $\mathbf{S}$, and the other is to solve dictionary $\mathbf{D}$. The optimization problem of sparse coding $\mathrm{S}$ is

$$
\min _{D, S}\|\mathbf{Y}-\mathbf{D S}\|_{F}^{2}+\lambda\left\|s_{i}\right\|_{1}, i=1, \ldots, n
$$

And the optimization problem of dictionary $\mathbf{D}$ is

$$
\begin{aligned}
& \min _{D, S}\|\mathbf{Y}-\mathbf{D S}\|_{F}^{2}, \\
& \text { s.t. }\left\|d_{j}\right\|_{2}^{2}<1, j=1, \ldots, K
\end{aligned}
$$

By alternately optimizing the above problems until convergence, the optimal $\mathbf{D}$ and $\mathbf{S}$ can be obtained. For the sample $\mathbf{y}$ on the test set, the corresponding sparse coding can be obtained by solving Equation 4, and then the classification is performed using the obtained sparse coding.

\section{TRANSFER LEARNING DISCRIMINATIVE DICTIONARY LEARNING ALGORITHM}

\section{Objective Function}

It is known that when there are few domain-invariant features between different domains, the cross-domain classification performance will decline. To extract as sufficient domaininvariant features as possible and reduce their differences between different domains, the TLDDL algorithm uses the dictionary learning model to learn a domain-invariant dictionary as a bridge to realize the association of the two domains. The source domain is $\mathbf{Y}_{s}=\left\{\mathbf{y}_{s, 1}, \mathbf{y}_{s, 2}, \ldots, \mathbf{y}_{s, n_{s}}\right\}$, and the target domain is $\mathbf{Y}_{t}=\left\{\mathbf{y}_{t, 1}, \mathbf{y}_{t, 2}, \ldots, \mathbf{y}_{t, n_{t}}\right\}$, where $n_{s}$ and $n_{t}$ are the number of the source domain and the target domain, respectively.

Firstly, I use the $k$-nearest neighbor knowledge to represent the local structure and label information of the original data. I think that if the sample $\mathbf{y}_{j}$ is in the $k$-nearest neighbor of its same-class sample $\mathbf{y}_{i}$, then the corresponding sparse coding coefficients $\mathbf{s}_{i}$ and $\mathbf{s}_{j}$ for $\mathbf{y}_{i}$ and $\mathbf{y}_{j}$ should also be closer to each other. On the other hand, it is necessary to minimize the withinclass variance and maximize the between-class separability for the sparse coding coefficients.

Given the training samples $\mathbf{y}_{i}$ and $\mathbf{y}_{j}$, the following withinclass graph matrix $E^{\text {wit }}$ and between-class graph matrix $\mathbf{E}_{s}^{\text {bet }}$ are defined as

$$
\begin{array}{r}
e_{i j}^{\text {wit }}= \begin{cases}e\left(\mathbf{y}_{i}, \mathbf{y}_{j}\right), & \text { if } \mathbf{y}_{i} \in N_{\text {wit }}\left(\mathbf{y}_{j}\right) \text { or } \mathbf{y}_{j} \in N_{\text {wit }}\left(\mathbf{y}_{i}\right) \\
0, & \text { otherwise }\end{cases} \\
e_{i j}^{\text {bet }}= \begin{cases}-e\left(\mathbf{y}_{i}, \mathbf{y}_{j}\right), & \text { if } \mathbf{y}_{i} \in N_{\text {bet }}\left(\mathbf{y}_{j}\right) \text { or } \mathbf{y}_{j} \in N_{\text {bet }}\left(\mathbf{y}_{i}\right) \\
0, & \text { otherwise }\end{cases}
\end{array}
$$

where $e\left(\mathbf{y}_{i}, \mathbf{y}_{j}\right)=\exp \left(-\left\|\mathbf{y}_{i}-\mathbf{y}_{j}\right\|^{2} / \theta\right), \theta$ is the tune parameter. $N_{\text {wit }}\left(\mathbf{y}_{i}\right)$ is the $k$-nearest neighbors of $\mathbf{y}_{i}$ belonging to the same class, and $N_{\text {bet }}\left(\mathbf{y}_{i}\right)$ is the $k$-nearest neighbors of $\mathbf{y}_{i}$ belonging to the different class. 
Considering the within-class minimizing and between-class maximizing of sparse coding coefficients, I define the following discrimination information preserved term $F_{1}\left(\mathbf{S}_{t}\right)$ in the source domain,

$$
\begin{aligned}
F_{1}\left(\mathbf{S}_{s}\right) & =\min _{\mathbf{S}_{s}} \frac{1}{2} \sum_{i=1}^{n_{s}} \sum_{j=1}^{n_{s}}\left\|\mathbf{s}_{s, i}-\mathbf{s}_{s, j}\right\|_{2}^{2}\left(\mathbf{E}_{s}^{\text {wit }}-\mathbf{E}_{s}^{b e t}\right) \\
& =\operatorname{Tr}\left(\mathbf{S}_{s} \mathbf{L}_{s}^{\text {wit }} \mathbf{S}_{s}^{T}\right)-\operatorname{Tr}\left(\mathbf{S}_{s} \mathbf{L}_{s}^{\text {bet }} \mathbf{S}_{s}^{T}\right) \\
& =\operatorname{Tr}\left(\mathbf{S}_{s} \mathbf{Q}_{s} \mathbf{S}_{s}^{T}\right),
\end{aligned}
$$

where $\mathbf{Q}_{s}=\mathbf{L}_{s}^{\text {wit }}-\mathbf{L}_{s}^{\text {bet }}, \operatorname{Tr}(\cdot)$ is the trace operator.

Similarly, I can obtain the discrimination information preserved term $F_{1}\left(S_{t}\right)$ in the target domain,

$$
\begin{aligned}
F_{1}\left(\mathbf{S}_{t}\right) & =\min _{\mathbf{S}_{t}} \frac{1}{2} \sum_{i=1}^{n_{t}} \sum_{j=1}^{n_{t}}\left\|\mathbf{s}_{t, i}-\mathbf{s}_{t, j}\right\|_{2}^{2}\left(\mathbf{E}_{t}^{\text {wit }}-\mathbf{E}_{t}^{\text {bet }}\right) \\
& =\operatorname{Tr}\left(\mathbf{S}_{t} \mathbf{L}_{t}^{\text {wit }} \mathbf{S}_{t}^{T}\right)-\operatorname{Tr}\left(\mathbf{S}_{t} \mathbf{L}_{t}^{\text {bet }} \mathbf{S}_{t}^{T}\right) \\
& =\operatorname{Tr}\left(\mathbf{S}_{t} \mathbf{Q}_{t} \mathbf{S}_{t}^{T}\right)
\end{aligned}
$$

where $\mathbf{Q}_{t}=\mathbf{L}_{t}^{\text {wit }}-\mathbf{L}_{t}^{\text {bet }}$.

When reconstructing the projection space, the proposed transfer learning algorithm not only needs to establish potential connections between multiple domains in the projection space, but also transfers the domain-invariant information from the source domain to the target domain. Meanwhile, TLDDL should also retain the identification information of label samples and maintain the discriminative information in the projection space. To achieve this goal, I establish the principal component analysis (PCA) term of the projection matrix. Based on the PCA criterion, the discrimination information in the original space will be retained in the projection space. The PCA terms of the projection matrix on the source and target domains are represented as

$$
\begin{aligned}
& F_{2}\left(\mathbf{P}_{s}\right)=\max _{\mathbf{P}_{s}} \operatorname{Tr}\left(\mathbf{P}_{s} \mathbf{Y}_{s} \mathbf{Y}_{s}^{T} \mathbf{P}_{s}^{T}\right), \\
& F_{2}\left(\mathbf{P}_{t}\right)=\max _{\mathbf{P}_{t}} \operatorname{Tr}\left(\mathbf{P}_{t} \mathbf{Y}_{t} \mathbf{Y}_{t}^{T} \mathbf{P}_{t}^{T}\right),
\end{aligned}
$$

where $\mathbf{P}_{s}, \mathbf{P}_{t} \in \mathbf{R}^{p \times d}, p$ and $d$ are the dimensions of the projection subspace and original feature space, respectively.

Based on the traditional dictionary learning algorithm, TLDDL combined the discriminative information preserved terms and PCA terms of the projection matrix together, and builds a domain-invariant dictionary between different domains. The objective function of TLDDL is

$$
\begin{aligned}
& \min _{D, P_{s}, P_{t}, S_{s}, S_{t}}\left\|P_{s} Y_{s}^{T}-D S_{s}\right\|_{2}^{2}+\left\|P_{t} Y_{t}^{T}-D S_{t}\right\|_{2}^{2}+\delta \operatorname{Tr}\left(S_{s} Q_{s} S_{s}^{T}\right) \\
&+\delta \operatorname{Tr}\left(S_{t} Q_{t} S_{t}^{T}\right) \\
&-\gamma \operatorname{Tr}\left(P_{s} Y_{s} Y_{s}^{T} P_{s}^{T}\right)-\gamma \operatorname{Tr}\left(P_{t} Y_{t} Y_{t}^{T} P_{t}^{T}\right)+\beta\left\|S_{s}\right\|_{2}^{2} \\
&+\beta\left\|S_{t}\right\|_{2}^{2} \\
& \text { s.t. }\left\|d_{i}\right\|_{2}^{2} \leq 1 \\
& P_{s}^{T} P_{s}=I \\
& P_{t}^{T} P_{t}=I
\end{aligned}
$$

where $\delta, \gamma$ and $\beta$ are trade off parameters.

For Equation 12, the first and second terms inherit the dictionary learning algorithm and are used to reconstruct the data in the source and target domains. These two terms are also domain-invariant dictionary learning terms to realize the

\begin{tabular}{|c|c|c|c|c|c|c|c|}
\hline Tasks & K-SVD & ARTL & DMTTL & SMITL & WAAR & SFA & TLDDL \\
\hline Sto $\rightarrow$ Cul & 62.31 & 66.18 & 66.64 & 66.35 & 68.89 & 68.63 & 70.64 \\
\hline Sto $\rightarrow$ Ent & 77.69 & 80.02 & 81.01 & 81.14 & 83.03 & 83.59 & 85.58 \\
\hline $\mathrm{Cul} \rightarrow$ Ent & 77.05 & 80.54 & 80.90 & 81.55 & 82.03 & 83.06 & 85.05 \\
\hline $\mathrm{Cul} \rightarrow \mathrm{Edu}$ & 78.72 & 82.37 & 81.39 & 83.85 & 84.87 & 84.79 & 86.76 \\
\hline $\mathrm{Ent} \rightarrow \mathrm{Spo}$ & 85.07 & 89.34 & 88.40 & 90.99 & 91.85 & 91.98 & 93.89 \\
\hline Ent $\rightarrow$ Edu & 77.15 & 80.13 & 81.08 & 81.91 & 83.99 & 83.59 & 85.51 \\
\hline Spo $\rightarrow$ Wor & 64.00 & 67.62 & 67.64 & 68.08 & 69.06 & 70.01 & 72.01 \\
\hline $\mathrm{Spo} \rightarrow \mathrm{Gam}$ & 82.04 & 85.46 & 85.02 & 86.37 & 87.86 & 88.02 & 90.03 \\
\hline Fin $\rightarrow$ Car & 85.08 & 88.59 & 88.59 & 89.94 & 90.15 & 91.12 & 93.02 \\
\hline Fin $\rightarrow$ Agr & 78.33 & 81.89 & 82.08 & 81.76 & 83.22 & 84.40 & 86.42 \\
\hline $\mathrm{Hou} \rightarrow$ Wor & 63.11 & 67.29 & 67.67 & 67.15 & 69.13 & 69.72 & 71.77 \\
\hline $\mathrm{Hou} \rightarrow$ Fin & 58.04 & 60.29 & 61.13 & 62.08 & 63.71 & 63.93 & 66.05 \\
\hline $\mathrm{Car} \rightarrow \mathrm{Tec}$ & 79.18 & 83.13 & 83.40 & 84.84 & 85.00 & 85.82 & 87.87 \\
\hline $\mathrm{Car} \rightarrow \mathrm{Gam}$ & 82.28 & 85.78 & 86.82 & 87.61 & 87.33 & 88.22 & 90.21 \\
\hline $\mathrm{Edu} \rightarrow \mathrm{Tec}$ & 79.02 & 82.14 & 82.89 & 83.78 & 84.04 & 85.34 & 87.32 \\
\hline Edu $\rightarrow$ Sto & 61.29 & 65.54 & 65.91 & 65.00 & 67.70 & 67.45 & 69.43 \\
\hline Tec $\rightarrow$ Wor & 63.30 & 67.32 & 67.56 & 68.43 & 69.47 & 69.82 & 71.87 \\
\hline $\mathrm{Tec} \rightarrow \mathrm{Mil}$ & 78.07 & 81.65 & 81.09 & 82.97 & 83.28 & 84.10 & 86.02 \\
\hline $\mathrm{Mil} \rightarrow$ Sto & 60.19 & 64.39 & 64.85 & 65.04 & 66.93 & 66.85 & 68.97 \\
\hline $\mathrm{Mil} \rightarrow$ Agr & 75.10 & 79.58 & 79.28 & 80.94 & 81.16 & 82.05 & 84.05 \\
\hline Wor $\rightarrow$ Mil & 78.60 & 81.21 & 82.92 & 83.97 & 84.67 & 84.67 & 86.66 \\
\hline Wor $\rightarrow$ Cul & 63.06 & 66.65 & 66.12 & 67.65 & 68.60 & 69.00 & 71.03 \\
\hline Agr $\rightarrow$ Fin & 58.29 & 62.91 & 62.06 & 63.74 & 64.18 & 64.76 & 66.78 \\
\hline Agr $\rightarrow$ Sto & 60.29 & 64.39 & 64.18 & 65.98 & 66.10 & 66.87 & 68.88 \\
\hline Gam $\rightarrow$ Spo & 85.05 & 89.56 & 89.93 & 89.10 & 91.72 & 91.43 & 93.47 \\
\hline Gam $\rightarrow$ Car & 84.17 & 88.07 & 88.85 & 89.80 & 90.94 & 90.92 & 92.79 \\
\hline Mean & 72.94 & 76.62 & 76.82 & 77.69 & 78.81 & 79.24 & 81.23 \\
\hline
\end{tabular}
connection of knowledge between different domains. The third and fourth terms are discriminative information preserved terms. The fifth and sixth terms are PCA terms of the projection matrices. The last two terms are the regularization terms of the sparse coding matrices.

\section{Optimization}

Let $\mathbf{P}=\left[\mathbf{P}_{s}, \mathbf{P}_{t}\right], \mathbf{Y}=\left[\begin{array}{cc}\mathbf{Y}_{s}^{T} & \mathbf{0} \\ \mathbf{0} & \mathbf{Y}_{t}^{T}\end{array}\right], \mathbf{S}=\left[\begin{array}{cc}\mathbf{S}_{s} & \mathbf{0} \\ \mathbf{0} & \mathbf{S}_{t}\end{array}\right], \mathbf{Q}=\left[\begin{array}{cc}\mathbf{Q}_{s} & \mathbf{0} \\ \mathbf{0} & \mathbf{Q}_{t}\end{array}\right]$, Equation 12 can be simplified as,

$$
\begin{aligned}
& \min _{\mathbf{D}, \mathbf{P}, \mathbf{S}}\left\|\mathbf{P Y} \mathbf{Y}^{T}-\mathbf{D S}\right\|_{F}^{2}+\operatorname{Tr}\left(\mathbf{S}(\delta \mathbf{Q}+\beta \mathbf{I}) \mathbf{S}^{T}\right)-\gamma \operatorname{Tr}\left(\mathbf{P Y} \mathbf{Y}^{T} \mathbf{P}^{T}\right), \\
& \text { s.t. }\left\|\mathbf{d}_{i}\right\|_{2}^{2} \leq 1, \quad \forall i \\
& \quad \mathbf{P}^{T} \mathbf{P}=\mathbf{I},
\end{aligned}
$$

The alternating iteration method is used to optimize variables $\{\mathbf{P}, \mathbf{S}, \mathbf{D}\}$. In the optimization process, other variables are fixed and only one variable is optimized.

TABLE 1 | Accuracy results for each cross-domain task on Chinese corpus.

The best classification results are indicated in bold in Table. 
Fixed $\{\mathbf{S}, \mathbf{D}\}$, the optimization problem of parameter $\mathbf{P}$ can be written as,

$$
\begin{aligned}
& \underset{P}{\min _{P} \| \mathbf{P Y}} \mathbf{Y}^{T}-\mathbf{D S} \|_{F}^{2}-\gamma \operatorname{Tr}\left(\mathbf{P Y Y} \mathbf{Y}^{T} \mathbf{P}^{T}\right), \\
& \text { s.t. } \mathbf{P} \mathbf{P}^{T}=\mathbf{I} .
\end{aligned}
$$

Following the Proposition (2) in Shekhar et al. (2013), let

$$
\begin{gathered}
\mathbf{P}=(\mathbf{Y W})^{T}, \\
\mathbf{D}=\mathbf{P Y C},
\end{gathered}
$$

where $\mathbf{W} \in \mathbf{R}^{\left(n_{s}+n_{t}\right) \times p}, \mathbf{C} \in \mathbf{R}^{\left(n_{s}+n_{t}\right) \times K}$.

Equation 14 can be written as,

$$
\begin{aligned}
& \underset{\mathbf{W}}{\min }\left\|\mathbf{W}^{T} \mathbf{M}(\mathbf{I}-\mathbf{C S})\right\|_{\mathbf{F}}^{2}-\gamma \operatorname{Tr}\left(\mathbf{W}^{T} \mathbf{M} \mathbf{M}^{T} \mathbf{W}\right), \\
& \text { s.t. } \mathbf{W} \mathbf{M} \mathbf{W}^{T}=\mathbf{I} .
\end{aligned}
$$

\begin{tabular}{|c|c|c|c|c|c|c|c|}
\hline Tasks & K-SVD & ARTL & DMTTL & SMITL & WAAR & SFA & TLDDL \\
\hline Stor $\rightarrow$ Cul & & & & & & 74.64 & \\
\hline Stor $\rightarrow$ Ent & & & & & & & \\
\hline $\mathrm{Cul} \rightarrow$ Ent & & & & & & & 85.37 \\
\hline $\mathrm{Cul} \rightarrow \mathrm{Edu}$ & & & & & & & 84.44 \\
\hline Ent $\rightarrow$ Spo & 85.15 & & & & & & \\
\hline Ent $\rightarrow$ Edu & & & & & & & 83.41 \\
\hline Spo $\rightarrow$ Wor & 64.07 & 67.61 & & & & & \\
\hline $\mathrm{Spo} \rightarrow \mathrm{Gam}$ & & & & & & & 92.35 \\
\hline Fin $\rightarrow$ Car & 85.29 & 88.53 & 88.48 & & & 91.01 & 93.22 \\
\hline Fin $\rightarrow$ Agr & & & & & 83.2 & & 86.38 \\
\hline $\mathrm{Hou} \rightarrow$ Wor & 63.03 & 67.21 & & 67.21 & & 69.79 & 71.81 \\
\hline $\mathrm{Hou} \rightarrow$ Fin & 57.99 & 60.35 & 61.15 & 62.14 & 63.57 & 63.92 & 66.03 \\
\hline $\mathrm{Car} \rightarrow \mathrm{Tec}$ & 79.01 & 83.19 & 83.41 & & & & 87.70 \\
\hline $\mathrm{Car} \rightarrow \mathrm{Gam}$ & 84.34 & 87.67 & & 89.78 & 89.4 & 36 & 92.15 \\
\hline $\mathrm{Edu} \rightarrow \mathrm{Tec}$ & 78.95 & 82.21 & & & & & 87.50 \\
\hline Edu $\rightarrow$ Stor & 59.32 & 63.46 & 63.67 & 62.76 & 65.8 & 65.47 & 67.20 \\
\hline Tec $\rightarrow$ Wor & 63.17 & 67.02 & 67.58 & 68.28 & & 70.01 & 71.71 \\
\hline $\mathrm{Tec} \rightarrow \mathrm{Mil}$ & 81.03 & 84.71 & 84.17 & 85.94 & 86.34 & 87.14 & 89.17 \\
\hline $\mathrm{Mil} \rightarrow$ Sto & 58.34 & 62.22 & 62.95 & 63.25 & & 64.84 & 67.03 \\
\hline $\mathrm{Mil} \rightarrow \mathrm{Agr}$ & 75.16 & 79.26 & 78.96 & 80.94 & 80.95 & 82.03 & 83.87 \\
\hline Wor $\rightarrow$ Mil & 81.47 & 84.09 & 85.92 & 87.06 & 87.61 & 87.65 & 89.88 \\
\hline Wor $\rightarrow$ Cul & 62.96 & 66.71 & & 67.53 & 68.87 & 68.95 & 71.28 \\
\hline Agr $\rightarrow$ Fin & 58.43 & 62.96 & 61.95 & & 64.40 & 64.94 & 67.02 \\
\hline Agr $\rightarrow$ Sto & 60.14 & 64.39 & & & 65.94 & 66.74 & 68.88 \\
\hline Gam $\rightarrow$ Spo & 85.05 & 89.45 & 89.71 & 89.01 & & 91.61 & 93.27 \\
\hline Gam $\rightarrow$ Car & 84.10 & 88.10 & & 89.82 & 90.86 & 90.81 & 92.96 \\
\hline Mean & 73.23 & 76.88 & 77.11 & 78.00 & 79.14 & 79.58 & 81.57 \\
\hline
\end{tabular}

where $\mathbf{M}=\mathbf{Y}^{T} \mathbf{Y}$.

Then $\mathbf{W}$ has a closed-form solution as,

$$
\mathbf{W}=\Theta \mathbf{V}^{-1 / 2} \mathbf{U}^{T}
$$

TABLE 2 | Precision results for each cross-domain task on Chinese corpus.

The best classification results are indicated in bold in Table. where $\mathbf{M}=\Theta \mathbf{V} \Theta^{T}$, and $\mathbf{U}$ can be obtained as,

$$
\begin{aligned}
& \min _{\mathbf{U}} \operatorname{Tr}\left(\mathbf{U}^{T} \mathbf{H}^{T} U\right), \\
& \text { s.t. } \mathbf{U}^{T} \mathbf{U}=\mathbf{I},
\end{aligned}
$$

where $\mathbf{H}=\mathbf{V}^{1 / 2} \Theta^{T}\left((\mathbf{I}-\mathbf{C S})(\mathbf{I}-\mathbf{C S})^{T}-\gamma \mathbf{I}\right) \Theta \mathbf{V}^{1 / 2}$. Due to the orthonormality condition on $\mathbf{U}$, Equation 19 has a closed-form solution. Therefore, $\mathbf{P}$ can then be updated by Equation 15 .

Fixed $\{\mathbf{P}, \mathbf{S}\}$, the optimization problem of parameter $\mathbf{D}$ can be written as,

$$
\begin{aligned}
& \min _{D}\|\mathbf{P Y}-\mathbf{D S}\|_{\mathbf{F}}^{2}, \\
& \text { s.t. }\left\|\mathbf{d}_{i}\right\|_{2}^{2} \leq 1 .
\end{aligned}
$$

Using the Lagrange dual method, $\mathbf{D}$ can be obtained as,

$$
\mathbf{D}=\left(\mathbf{P Y S}^{T}\right)\left(\mathbf{S S}^{T}+\Delta\right)^{-1},
$$

where $\Delta$ is a diagonal matrix.

Fixed $\{\mathbf{P}, \mathbf{D}\}$, the optimization problem of parameter $\mathbf{S}$ can be written as,

$$
\min _{S}\left\|\mathbf{P} \mathbf{Y}^{T}-\mathbf{D S}\right\|_{\mathbf{F}}^{2}+\operatorname{Tr}\left(\mathbf{S}(\delta \mathbf{Q}+\beta \mathbf{I}) \mathbf{S}^{T}\right),
$$

By setting the derivative of $\mathbf{S}$ to zero, I get,

$$
\mathbf{D}^{T} \mathbf{D S}+\mathbf{S}(\delta \mathbf{Q}+\beta \mathbf{I})=\mathbf{D}^{T} \mathbf{P Y} .
$$

In this study, Equation 23 is solved by the Bartels-Stewart method (Kleinman and Rao, 2003).

The above optimization steps are summarized in Algorithm 1.

\section{ALGORITHM 1 | Algorithm 1 the TLDDL algorithm.}

Input: Training data $\mathbf{Y}_{s}$ and $\mathbf{Y}_{t}$;

Output: dictionary $\mathbf{D}$, projection matrix $\mathbf{P}_{S}$ and $\mathbf{P}_{t}$

Step 1. Initialize $\mathbf{D}$ and $\mathbf{S}$ using the K-SVD algorithm (Aharon et al., 2006);

Step 2. Compute the matrices $\mathbf{E}^{\text {wit }}$ and $\mathbf{E}_{S}^{\text {bet }}$ via Equation 6, 7;

Step 3. Learn $\mathbf{P}$ with fixed $\{\mathbf{S}, \mathbf{D}\}$ via Equations 14-19;

Step 4. Learn $\mathbf{D}$ with fixed $\{\mathbf{P}, \mathbf{S}\}$ via Equation 21;

Step 5. Learn $\mathbf{S}$ with fixed $\{\mathbf{P}, \mathbf{D}\}$ via Equation 23;

Step 6. Go to Step 3 until the convergence or reaching the maximum number of iterations

Step 7. Output the dictionary $\mathbf{D}$, projection matrix $\mathbf{P}_{s}$ and $\mathbf{P}_{t}$.

\section{Test}

For an unlabeled test text $\mathbf{y}^{\text {test }}$, according to the obtained optimal projection matrix $\mathbf{P}_{t}$ and dictionary $\mathbf{D}$, its sparse coding coefficient $\mathbf{s}^{*}$ can be solved by the following problem,

$$
\min _{\mathbf{s}^{*}}\left\|\mathbf{P}_{t}\left(\mathbf{y}^{\text {test }}\right)^{T}-\mathbf{D s}^{*}\right\|_{2}^{2}+\beta\left\|\mathbf{s}^{*}\right\|_{2}^{2} \text {. }
$$

I can obtain $\mathbf{s}^{*}$ as follows,

$$
\mathbf{s}^{*}=\left(\mathbf{D D}^{T}+\beta \mathbf{I}\right)^{-1} \mathbf{D}^{T} \mathbf{P}_{t} .
$$

Then, I compute the reconstruction error of $\mathbf{y}^{\text {test }}$ on the dictionary $D=\left[\mathbf{D}_{1}, \mathbf{D}_{2}, \ldots, \mathbf{D}_{J}\right]$, where $J$ is the number of sample 
classes. The reconstruction error of $\mathbf{y}^{\text {test }}$ on the $j$ th sub dictionary $\mathbf{D}_{j}$ can be computed as follows,

$$
\mathbf{r}_{j}\left(\mathbf{y}^{\text {test }}\right)=\left\|\mathbf{y}^{\text {test }}-\mathbf{D}_{j} \mathbf{s}_{j}^{*}\right\|_{2}^{2},
$$

where $\mathbf{s}_{j}^{*}$ is the sparse coding coefficient of $\mathbf{y}^{\text {test }}$ on the $D_{j}$. Finally, the class label of $\mathbf{y}^{\text {test }}$ is the class with the smallest reconstruction error, i.e.,

$$
\operatorname{label}\left(\mathbf{y}^{\text {test }}\right)=\min _{j} \mathbf{r}_{j}\left(\mathbf{y}^{\text {test }}\right) .
$$

\section{EXPERIMENT}

\section{Datasets and Experiment Setup}

The experiment adopts the Chinese corpus (including toutiaotext and Chinese Weibo datasets) to verify the proposed TLDDL algorithm. I select 13 categories in Chinese corpus includes: Story (Sto), Culture (Cul), Entertainment (Ent), Sports (Spo), Finance (Fin), House (Hou), Car, Education (Edu), Technology $(\mathrm{Tec})$, Military (Mil), World (Wor), Agriculture (Agr), and Game (Gam). Each category represents a domain. In addition, the experiment adopts the English multi-domain dataset Amazon review dataset, which is often used in cross-domain sentiment classification. Amazon review dataset includes reviews of DVD, book (Boo), electronic (Ele), kitchen and household appliance

TABLE 3 | Recall results for each cross-domain task on Chinese corpus.

\begin{tabular}{lccccccc}
\hline Tasks & K-SVD & ARTL & DMTTL & SMITL & WAAR & SFA & TLDDL \\
\hline Stor $\rightarrow$ Cul & 76.10 & 79.95 & 80.70 & 80.53 & 82.62 & 82.69 & $\mathbf{8 4 . 6 1}$ \\
Stor $\rightarrow$ Ent & 76.63 & 79.09 & 80.00 & 80.12 & 82.04 & 82.56 & $\mathbf{8 4 . 4 3}$ \\
Cul $\rightarrow$ Ent & 75.99 & 79.63 & 79.75 & 80.56 & 80.74 & 81.87 & $\mathbf{8 4 . 2 8}$ \\
Cul $\rightarrow$ Edu & 77.62 & 81.38 & 80.41 & 82.59 & 83.92 & 83.91 & $\mathbf{8 5 . 5 8}$ \\
Ent $\rightarrow$ Spo & 83.88 & 88.55 & 87.18 & 89.76 & 90.74 & 91.10 & $\mathbf{9 2 . 8 6}$ \\
Ent $\rightarrow$ Edu & 76.28 & 79.21 & 80.30 & 80.84 & 82.79 & 82.62 & $\mathbf{8 4 . 4 9}$ \\
Spo $\rightarrow$ Wor & 61.09 & 64.63 & 64.43 & 65.19 & 66.20 & 66.85 & $\mathbf{6 9 . 2 5}$ \\
Spo $\rightarrow$ Gam & 77.01 & 80.38 & 80.06 & 81.25 & 83.13 & 82.95 & $\mathbf{8 5 . 1 6}$ \\
Fin $\rightarrow$ Car & 84.25 & 87.40 & 87.54 & 89.15 & 88.98 & 90.17 & $\mathbf{9 2 . 0 6}$ \\
Fin $\rightarrow$ Agr & 77.36 & 80.86 & 81.11 & 80.76 & 82.14 & 83.56 & $\mathbf{8 5 . 4 6}$ \\
Hou $\rightarrow$ Wor & 62.23 & 66.47 & 66.83 & 66.16 & 68.21 & 68.91 & $\mathbf{7 0 . 5 3}$ \\
Hou $\rightarrow$ Fin & 57.76 & 60.59 & 61.32 & 62.32 & 63.70 & 64.13 & $\mathbf{6 6 . 1 1}$ \\
Car $\rightarrow$ Tec & 77.91 & 82.43 & 82.39 & 83.81 & 83.97 & 84.95 & $\mathbf{8 6 . 6 2}$ \\
Car $\rightarrow$ Gam & 77.01 & 80.72 & 81.74 & 82.68 & 82.43 & 82.99 & $\mathbf{8 5 . 0 4}$ \\
Edu $\rightarrow$ Tec & 77.97 & 81.22 & 81.87 & 82.57 & 82.90 & 84.04 & $\mathbf{8 6 . 3 9}$ \\
Edu $\rightarrow$ Stor & 63.28 & 67.53 & 67.81 & 67.26 & 69.59 & 69.59 & $\mathbf{7 1 . 5 4}$ \\
Tec $\rightarrow$ Wor & 60.30 & 64.38 & 64.74 & 65.32 & 66.53 & 66.89 & $\mathbf{6 8 . 7 0}$ \\
Tec $\rightarrow$ Mil & 74.04 & 77.75 & 77.00 & 79.16 & 79.47 & 80.37 & $\mathbf{8 1 . 9 7}$ \\
Mil $\rightarrow$ Sto & 61.94 & 66.26 & 66.68 & 67.17 & 68.95 & 68.81 & $\mathbf{7 1 . 1 8}$ \\
Mil $\rightarrow$ Agr & 74.02 & 78.74 & 78.24 & 79.96 & 80.04 & 81.32 & $\mathbf{8 2 . 7 8}$ \\
Wor $\rightarrow$ Mil & 74.45 & 77.42 & 79.09 & 80.00 & 80.47 & 80.61 & $\mathbf{8 2 . 7 5}$ \\
Wor $\rightarrow$ Cul & 77.13 & 80.56 & 80.10 & 81.83 & 82.70 & 83.04 & $\mathbf{8 5 . 0 2}$ \\
Agr $\rightarrow$ Fin & 58.52 & 63.11 & 61.89 & 63.92 & 64.17 & 64.64 & $\mathbf{6 6 . 8 2}$ \\
Agr $\rightarrow$ Sto & 59.41 & 63.66 & 63.29 & 64.99 & 65.33 & 65.84 & $\mathbf{6 7 . 7 7}$ \\
Gam $\rightarrow$ Spo & 83.90 & 88.56 & 88.85 & 87.92 & 90.78 & 90.26 & $\mathbf{9 2 . 3 6}$ \\
Gam $\rightarrow$ Car & 83.24 & 87.01 & 87.65 & 88.86 & 90.07 & 89.83 & $\mathbf{9 1 . 8 7}$ \\
Mean & 72.67 & 76.44 & 76.58 & 77.49 & 78.56 & 79.02 & $\mathbf{8 0 . 9 9}$ \\
\hline Th $b$ St & & & & & & &
\end{tabular}

The best classification results are indicated in bold in Table.
(Kit). Each product also represents a domain. To facilitate comparison with existing methods, 2,000 comments are selected respectively, including 1,000 positive comments and 1,000 negative comments. I use $80 \%$ texts in the source domain and $10 \%$ texts in the target domain as the training dataset, and the rest of the texts in the target domain are used for testing.

I investigate the performance of TLDDL compared with several algorithms on two text corpora. For comparison, the K-SVD algorithm (Aharon et al., 2006) is used as the baseline

TABLE 4 | F1-score results for each cross-domain task on Chinese corpus.

\begin{tabular}{lccccccc}
\hline Tasks & K-SVD & ARTL & DMTTL & SMITL & WAAR & SFA & TLDDL \\
\hline Stor $\rightarrow$ Cul & 72.50 & 75.91 & 76.44 & 76.41 & 79.09 & 78.62 & $\mathbf{8 0 . 5 5}$ \\
Stor $\rightarrow$ Ent & 77.41 & 79.44 & 80.54 & 80.62 & 82.76 & 83.06 & $\mathbf{8 4 . 7 6}$ \\
Cul $\rightarrow$ Ent & 76.23 & 79.90 & 80.44 & 80.93 & 82.03 & 82.48 & $\mathbf{8 4 . 7 0}$ \\
Cul $\rightarrow$ Edu & 77.26 & 80.97 & 80.19 & 82.34 & 83.26 & 83.34 & $\mathbf{8 4 . 6 6}$ \\
Ent $\rightarrow$ Spo & 84.53 & 88.76 & 87.83 & 89.94 & 91.34 & 91.97 & $\mathbf{9 3 . 4 8}$ \\
Ent $\rightarrow$ Edu & 75.83 & 78.98 & 79.86 & 80.40 & 82.17 & 81.77 & $\mathbf{8 4 . 2 2}$ \\
Spo $\rightarrow$ Wor & 62.63 & 66.18 & 65.75 & 66.31 & 67.89 & 68.52 & $\mathbf{7 0 . 8 5}$ \\
Spo $\rightarrow$ Gam & 80.71 & 83.54 & 83.34 & 84.83 & 86.72 & 86.40 & $\mathbf{8 8 . 4 5}$ \\
Fin $\rightarrow$ Car & 84.83 & 87.72 & 87.91 & 89.61 & 89.69 & 90.53 & $\mathbf{9 2 . 6 8}$ \\
Fin $\rightarrow$ Agr & 78.08 & 81.22 & 81.50 & 81.19 & 82.59 & 84.54 & $\mathbf{8 6 . 0 2}$ \\
Hou $\rightarrow$ Wor & 62.93 & 67.18 & 66.99 & 66.93 & 68.53 & 69.51 & $\mathbf{7 1 . 0 1}$ \\
Hou $\rightarrow$ Fin & 57.92 & 60.16 & 61.20 & 62.57 & 63.61 & 63.70 & $\mathbf{6 6 . 0 5}$ \\
Car $\rightarrow$ Tec & 78.47 & 83.20 & 83.05 & 84.10 & 84.37 & 85.68 & $\mathbf{8 6 . 7 5}$ \\
Car $\rightarrow$ Gam & 80.37 & 84.15 & 85.17 & 86.03 & 86.00 & 86.85 & $\mathbf{8 8 . 8 1}$ \\
Edu $\rightarrow$ Tec & 78.88 & 81.77 & 82.27 & 82.99 & 83.17 & 84.90 & $\mathbf{8 6 . 9 3}$ \\
Edu $\rightarrow$ Stor & 61.08 & 65.37 & 65.34 & 64.68 & 67.43 & 67.60 & $\mathbf{6 9 . 3 8}$ \\
Tec $\rightarrow$ Wor & 61.85 & 65.72 & 65.87 & 66.72 & 68.24 & 68.40 & $\mathbf{7 0 . 3 3}$ \\
Tec $\rightarrow$ Mil & 77.28 & 81.46 & 80.43 & 82.39 & 82.87 & 83.94 & $\mathbf{8 5 . 7 3}$ \\
Mil $\rightarrow$ Sto & 60.14 & 64.29 & 64.76 & 64.87 & 66.86 & 66.75 & $\mathbf{6 9 . 2 2}$ \\
Mil $\rightarrow$ Agr & 74.49 & 78.99 & 78.66 & 80.76 & 80.56 & 81.64 & $\mathbf{8 3 . 0 1}$ \\
Wor $\rightarrow$ Mil & 77.81 & 80.48 & 82.72 & 83.75 & 83.89 & 84.24 & $\mathbf{8 6 . 4 1}$ \\
Wor $\rightarrow$ Cul & 69.84 & 73.58 & 72.86 & 74.60 & 75.94 & 75.60 & $\mathbf{7 8 . 2 5}$ \\
Agr $\rightarrow$ Fin & 58.47 & 63.05 & 61.76 & 63.93 & 64.05 & 64.87 & $\mathbf{6 7 . 0 3}$ \\
Agr $\rightarrow$ Sto & 59.67 & 64.21 & 63.51 & 65.59 & 65.88 & 66.34 & $\mathbf{6 8 . 4 4}$ \\
Gam $\rightarrow$ Spo & 84.18 & 89.08 & 88.98 & 88.52 & 91.51 & 90.65 & $\mathbf{9 3 . 0 7}$ \\
Gam $\rightarrow$ Car & 83.66 & 87.58 & 88.21 & 89.37 & 90.50 & 90.26 & $\mathbf{9 2 . 2 2}$ \\
Mean & 72.96 & 76.65 & 76.75 & 77.71 & 78.88 & 79.31 & $\mathbf{8 1 . 2 7}$ \\
\hline & & & & & & &
\end{tabular}

The best classification results are indicated in bold in Table.

TABLE 5 | Accuracy results for each cross-domain task on English corpus.

\begin{tabular}{lccccccc}
\hline Tasks & K-SVD & ARTL & DMTTL & SMITL & WAAR & SFA & TLDDL \\
\hline DVD $\rightarrow$ Boo & 74.44 & 76.76 & 77.18 & 78.00 & 79.95 & 81.05 & $\mathbf{8 2 . 9 8}$ \\
DVD $\rightarrow$ Ele & 70.28 & 72.44 & 73.30 & 73.68 & 74.65 & 76.01 & $\mathbf{7 6 . 7 8}$ \\
DVD $\rightarrow$ Kit & 71.09 & 73.70 & 73.79 & 74.92 & 75.03 & 75.67 & $\mathbf{7 6 . 0 9}$ \\
Boo $\rightarrow$ DVD & 76.32 & 79.02 & 79.20 & 81.02 & 82.01 & 82.94 & $\mathbf{8 4 . 7 4}$ \\
Boo $\rightarrow$ Ele & 71.32 & 73.41 & 74.12 & 75.52 & 76.29 & 77.28 & $\mathbf{7 8 . 0 7}$ \\
Boo $\rightarrow$ Kit & 73.64 & 76.36 & 76.64 & 78.13 & 77.43 & 77.67 & $\mathbf{7 8 . 1 2}$ \\
Ele $\rightarrow$ DVD & 73.04 & 75.78 & 75.96 & 77.56 & 79.02 & 80.54 & $\mathbf{8 1 . 6 3}$ \\
Ele $\rightarrow$ Boo & 70.88 & 73.88 & 73.34 & 75.68 & 76.67 & 78.66 & $\mathbf{8 0 . 3 3}$ \\
Ele $\rightarrow$ Kit & 83.23 & 85.61 & 86.32 & 87.71 & 85.39 & 86.37 & $\mathbf{8 7 . 5 4}$ \\
Kit $\rightarrow$ DVD & 74.73 & 77.44 & 77.04 & 78.77 & 79.27 & 80.14 & $\mathbf{8 0 . 7 8}$ \\
Kit $\rightarrow$ Boo & 82.07 & 84.53 & 85.11 & 86.03 & 86.02 & 86.90 & $\mathbf{8 7 . 2 8}$ \\
Kit $\rightarrow$ Ele & 73.65 & 75.95 & 76.59 & 77.57 & 78.88 & 79.67 & $\mathbf{8 0 . 7 6}$ \\
Mean & 74.56 & 77.07 & 77.38 & 78.72 & 79.22 & 80.24 & $\mathbf{8 1 . 2 6}$
\end{tabular}

The best classification results are indicated in bold in Table. 
TABLE 6 | Precision results for each cross-domain task on English corpus.

\begin{tabular}{lccccccc}
\hline Tasks & K-SVD & ARTL & DMTTL & SMITL & WAAR & SFA & TLDDL \\
\hline $\mathrm{DVD} \rightarrow$ Boo & 74.75 & 77.65 & 77.45 & 78.57 & 80.26 & 81.36 & $\mathbf{8 3 . 2 5}$ \\
$\mathrm{DVD} \rightarrow$ Ele & 71.03 & 73.02 & 73.82 & 73.96 & 75.28 & 76.16 & $\mathbf{7 7 . 1 2}$ \\
$\mathrm{DVD} \rightarrow$ Kit & 71.85 & 73.84 & 73.89 & 75.13 & 75.68 & 76.32 & $\mathbf{7 6 . 4 3}$ \\
$\mathrm{BoO} \rightarrow$ DVD & 76.51 & 79.88 & 79.45 & 81.02 & 82.76 & 83.71 & $\mathbf{8 5 . 4 2}$ \\
$\mathrm{BoO} \rightarrow$ Ele & 72.13 & 73.93 & 74.75 & 75.58 & 77.15 & 77.49 & $\mathbf{7 8 . 4 0}$ \\
$\mathrm{BoO} \rightarrow$ Kit & 73.96 & 76.83 & 77.02 & 78.26 & 77.82 & 78.57 & $\mathbf{7 8 . 6 1}$ \\
Ele $\rightarrow$ DVD & 73.15 & 75.80 & 76.16 & 77.77 & 79.85 & 81.16 & $\mathbf{8 1 . 8 0}$ \\
Ele $\rightarrow$ Boo & 71.60 & 74.16 & 73.75 & 75.98 & 77.42 & 78.97 & $\mathbf{8 0 . 9 0}$ \\
Ele $\rightarrow$ Kit & 83.78 & 85.68 & 86.84 & 88.21 & 85.73 & 87.06 & $\mathbf{8 8 . 3 9}$ \\
Kit $\rightarrow$ DVD & 75.53 & 77.81 & 77.28 & 79.26 & 79.39 & 80.85 & $\mathbf{8 1 . 3 0}$ \\
Kit $\rightarrow$ Boo & 82.58 & 84.90 & 85.62 & 86.77 & 86.54 & 87.26 & $\mathbf{8 7 . 6 7}$ \\
Kit $\rightarrow$ Ele & 73.80 & 76.73 & 77.05 & 78.31 & 79.60 & 79.86 & $\mathbf{8 0 . 7 7}$ \\
Mean & 75.06 & 77.52 & 77.76 & 79.07 & 79.79 & 80.73 & $\mathbf{8 1 . 6 7}$
\end{tabular}

The best classification results are indicated in bold in Table.

TABLE 7 | Recall results for each cross-domain task on English corpus.

\begin{tabular}{lccccccc}
\hline Tasks & K-SVD & ARTL & DMTTL & SMITL & WAAR & SFA & TLDDL \\
\hline $\mathrm{DVD} \rightarrow$ Boo & 73.86 & 76.23 & 77.14 & 77.30 & 79.93 & 80.44 & $\mathbf{8 2 . 6 9}$ \\
$\mathrm{DVD} \rightarrow$ Ele & 69.48 & 71.69 & 72.82 & 73.51 & 73.98 & 75.38 & $\mathbf{7 6 . 1 4}$ \\
$\mathrm{DVD} \rightarrow$ Kit & 70.87 & 72.95 & 73.70 & 74.20 & 74.43 & 75.03 & $\mathbf{7 6 . 0 4}$ \\
$\mathrm{BoO} \rightarrow$ DVD & 75.78 & 78.39 & 78.54 & 80.40 & 81.75 & 82.20 & $\mathbf{8 4 . 0 5}$ \\
$\mathrm{BoO} \rightarrow$ Ele & 71.18 & 73.21 & 73.93 & 74.77 & 76.05 & 77.06 & $\mathbf{7 7 . 5 7}$ \\
$\mathrm{BoO} \rightarrow$ Kit & 73.43 & 75.61 & 76.07 & 78.12 & 76.68 & 77.36 & $\mathbf{7 7 . 3 9}$ \\
Ele $\rightarrow$ DVD & 72.35 & 75.77 & 75.81 & 77.02 & 78.35 & 80.02 & $\mathbf{8 1 . 0 6}$ \\
Ele $\rightarrow$ Boo & 70.87 & 73.36 & 73.30 & 75.05 & 76.17 & 77.97 & $\mathbf{7 9 . 9 7}$ \\
Ele $\rightarrow$ Kit & 82.91 & 85.48 & 85.55 & 87.35 & 84.78 & 86.15 & $\mathbf{8 7 . 4 8}$ \\
Kit $\rightarrow$ DVD & 73.98 & 76.96 & 76.55 & 78.70 & 78.94 & 79.41 & $\mathbf{8 0 . 7 6}$ \\
Kit $\rightarrow$ Boo & 82.03 & 83.78 & 85.08 & 85.29 & 85.85 & 86.78 & $\mathbf{8 6 . 6 8}$ \\
Kit $\rightarrow$ Ele & 73.63 & 75.50 & 76.07 & 77.35 & 78.09 & 79.66 & $\mathbf{8 0 . 6 4}$ \\
Mean & 74.20 & 76.58 & 77.05 & 78.26 & 78.75 & 79.79 & $\mathbf{8 0 . 8 7}$
\end{tabular}

The best classification results are indicated in bold in Table.

of the proposed algorithm. The size of dictionary in K-SVD is set to 300. In addition, six transfer learning algorithms are used, including: ARTL (Long et al., 2014), DMTTL (Zheng et al., 2019), SMITL (Liu et al., 2018), WAAR (Jia et al., 2018), and SFA (Pan et al., 2010). In ARTL, the parameter $\lambda$ is set in the grid $\left\{10^{-2}, 10^{-1}, \ldots, 10^{2}\right\}$, the parameter $\gamma$ is set in the grid $\{0.01,0.05,0.1,0.5,1.5,10\}$, and the parameter $\sigma$ is set in the grid $\{0.01,0.02,0.1,0.5,1,2,5\}$. In DMTTL, the parameters $\lambda, \lambda_{s}, \lambda_{t}$ are set in the grid $\left\{10^{-4}, 10^{-3}, \ldots, 10^{3}\right\}, \gamma$ is set in the
TABLE 8 | F1-score results for each cross-domain task on English corpus.

\begin{tabular}{lccccccc}
\hline Tasks & K-SVD & ARTL & DMTTL & SMITL & WAAR & SFA & TLDDL \\
\hline $\mathrm{DVD} \rightarrow$ Boo & 74.08 & 76.90 & 77.49 & 78.03 & 80.13 & 80.85 & $\mathbf{8 2 . 8 9}$ \\
DVD $\rightarrow$ Ele & 70.08 & 72.18 & 73.25 & 73.96 & 74.70 & 75.72 & $\mathbf{7 6 . 5 8}$ \\
DVD $\rightarrow$ Kit & 71.54 & 73.13 & 73.66 & 75.07 & 74.81 & 75.71 & $\mathbf{7 6 . 5 6}$ \\
Boo $\rightarrow$ DVD & 76.05 & 79.53 & 78.79 & 80.89 & 82.42 & 82.84 & $\mathbf{8 4 . 8 3}$ \\
Boo $\rightarrow$ Ele & 71.25 & 73.94 & 74.43 & 75.42 & 76.43 & 77.37 & $\mathbf{7 7 . 9 7}$ \\
Boo $\rightarrow$ Kit & 73.69 & 75.84 & 76.58 & 78.37 & 77.18 & 77.68 & $\mathbf{7 7 . 9 8}$ \\
Ele $\rightarrow$ DVD & 72.62 & 75.97 & 75.92 & 77.24 & 79.11 & 80.51 & $\mathbf{8 1 . 4 0}$ \\
Ele $\rightarrow$ Boo & 71.16 & 73.39 & 73.77 & 75.17 & 76.50 & 78.57 & $\mathbf{7 9 . 9 6}$ \\
Ele $\rightarrow$ Kit & 83.05 & 85.39 & 86.34 & 87.63 & 84.83 & 86.56 & $\mathbf{8 8 . 0 9}$ \\
Kit $\rightarrow$ DVD & 74.77 & 77.37 & 76.77 & 78.98 & 79.37 & 80.09 & $\mathbf{8 0 . 9 6}$ \\
Kit $\rightarrow$ Boo & 81.86 & 84.19 & 85.30 & 86.36 & 86.36 & 87.12 & $\mathbf{8 7 . 2 9}$ \\
Kit $\rightarrow$ Ele & 74.06 & 76.26 & 76.83 & 77.50 & 79.01 & 79.94 & $\mathbf{8 0 . 5 0}$ \\
Mean & 74.52 & 77.01 & 77.43 & 78.72 & 79.24 & 80.25 & $\mathbf{8 1 . 2 5}$
\end{tabular}

The best classification results are indicated in bold in Table.

grid $\left\{10^{2}, 5 \times 10^{2}, \ldots, 2 \times 10^{4}\right\}$. In SMITL, the Gaussian kernel is used, and the kernel and penalty parameters are set in the grid $\left\{10^{-4}, 10^{-3}, \ldots, 10^{3}\right\}$. In WAAR, the parameter $l$ is set to 600 , min-support $=0.014$, min-confidence $=0.08$, and $\varepsilon=0.005$. In SFA, the parameters $l, k$ and $\gamma$ are set to 500, 100 and 0.6 , respectively. In the proposed TLDDL, the subspace dimension and the size of dictionary are set to 500 and 300 , respectively. The parameters $\delta_{s}, \delta_{t}, \gamma_{s}, \gamma_{t}, \beta_{s}$ and $\beta_{t}$ are set in the grid $\{0.01,0.05, \ldots$, $2\}$. In the experiments, the dimension of the text word vectors is set to 300. I take the first 100 text units in each text. Following (Meng et al., 2019), a convolutional neural network composed of five layers is used to extract the text features. The mini-batch size is set to 16 , and the number of convolution kernels is set to 256. Finally, these vectors are set to 768 dimensions when I treat them into the training model. All the experiments are executed in Matlab 2018a environment. I repeat the experiments five times. The performances of experimental results are generally evaluated based on accuracy, precision, recall, and F1-score.

\section{Experiments on Chinese Corpus}

Tables 1-4 show the classification results on Chinese corpus based on accuracy, precision, recall, and F1-score, respectively. For example, task "Stor $\rightarrow$ Cul" indicates that the source domain is "Story" and the target domain is "Culture." In the respect of classification accuracy, the best average accuracy obtained by
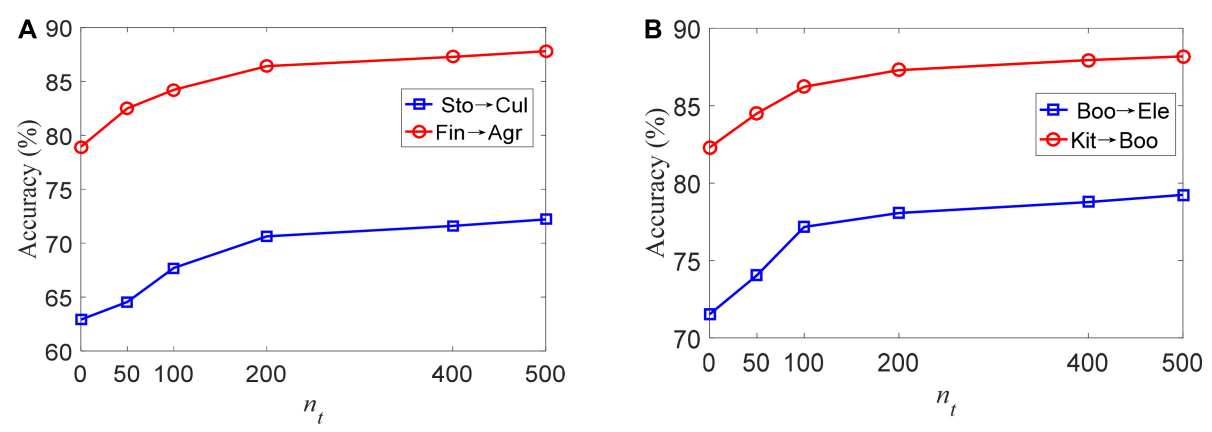

FIGURE 1 | The accuracy of TLDDL with different size of $\boldsymbol{n}_{\boldsymbol{t}}$, (A) Chinese corpus; (B) English corpus. 
TLDDL is $81.23 \%$, followed by SFA, and its average accuracy is $79.24 \%$. The non-transfer learning algorithm K-SVD is the lowest. In the respect of precision, recall, and F1-score, all transfer learning algorithms are always better than the non-transfer learning algorithm K-SVD in each classification task. The proposed TLDDL algorithm also obtains the best performance. Compared with the second-best algorithm, the average precision, recall, and F1 score exceed 1.99, 1.97, and $1.96 \%$, respectively. Therefore, the generalization ability of the proposed TLDDL algorithm is higher. The experimental results indicate that under the framework of the dictionary learning algorithm, TLDDL uses the projection technology to reduce the differences between different domains, and builds a domaininvariant dictionary to establish a bridge between the related domains. The transferring discriminative information of the source domain to the target domain can improve the crossdomain classification performance.

\section{Experiments on English Corpus}

Tables 5-8 show the classification results on the English corpus based on accuracy, precision, recall, and F1-score, respectively. I can see that all transfer learning algorithms outperform the baseline algorithm K-SVD. Some tasks have high classification performance, such as DVD $\rightarrow$ Boo and Boo $\rightarrow$ DVD. The reason for the high classification performance is that the differences between the source domain and the target domain are similar. Some tasks have low classification performance, such as DVD $\rightarrow$ Ele and DVD $\rightarrow$ Kit. The reason is that the domaininvariant information transferring from the source domain to the target domain is insufficient due to the great differences between domains. The results in Tables 5-8 show that the TLDDL algorithm obtains the best performance. TLDDL obtains the classification performance $81.26,81.67,80.87$, and $81.25 \%$ in the respect of accuracy, precision, recall and F1-score, respectively. TLDDL is $1.02,0.94,0.98$, and $1.00 \%$ higher than the second-best algorithm in four evaluation indexes. The results indicate that joint learning of projection technology and dictionary learning is an efficient strategy in cross-domain text classification tasks. In addition, the discrimination information preserved and PCA terms are helping to improve the generalization of the classifier.

\section{Experiments With Different Training Samples in the Target Domain}

To show the influence of the number of training samples in the target domain on the proposed TLDDL algorithm, I compare its classification accuracy on the Chinese and English corpora in Figure 1. The size of $n_{t}$ is set to be $0,50,100,200,400$, and 500, respectively. Since the size of $n_{t}$ should be less than that of $n_{s}$, the maximum size of $n_{t}$ is set to 500 . The results of Sto $\rightarrow \mathrm{Cul}$ and Fin $\rightarrow$ Agr on the Chinese corpus are shown in Figure 1A. When the size of $n_{t}$ is 0 , the classification accuracy of the two tasks is

\section{REFERENCES}

Aharon, M., Elad, M., and Bruckstein, A. (2006). K-SVD: an algorithm for designing overcomplete dictionaries for sparse representation. IEEE Trans. Signal Process. 54, 4311-4322. doi: 10.1109/TSP.2006.881199 the lowest. With the increase of the size of $n_{t}$, the classification accuracy is gradually improved. When the size of $n_{t}$ exceeds 200 , the classification accuracy reaches a relatively stable state. The results of $\mathrm{Boo} \rightarrow$ Ele and $\mathrm{Kit} \rightarrow \mathrm{Boo}$ on the English corpus are shown in Figure 1B. I can see that the classification accuracy of TLDDL increases rapidly after adding the training samples in $n_{t}$. When $n_{t}$ reaches 200 , the classification accuracy is stable and the growth rate of accuracy is small. Considering the practical application scenarios of transfer learning, 200 texts selected in the target domain for training are reasonable.

\section{CONCLUSION}

In this study, I have developed a transfer learning classification algorithm for cross-domain text sentiment classification. Inspired by the advantage of dictionary learning in knowledge reconstruction and sparse representation, I proposed to employ subspace projection and transfer learning into the framework of dictionary learning. Considering the within-class minimizing and between-class maximizing of sparse coding coefficients, I define the following discrimination information preserved term in the objective function; meanwhile, I adopt the PCA term in the objective function to retain the discrimination knowledge. In such an algorithm, a domain-invariant dictionary is built to establish a connection between different domains. Experimental results indicate that the TLDDL algorithm achieves good classification performance. In the future, from the perspective of multiple learning strategies on dictionary learning, I will consider how to realize transfer learning of multi-source and heterogeneous data in the proposed algorithm. In addition, from the perspective of extracting features, I will investigate how to automatically implement feature selection and building a classifier in a model framework.

\section{DATA AVAILABILITY STATEMENT}

Publicly available datasets were analyzed in this study. This data can be found here: The toutiao-text dataset is addressed as: https://github.com/aceimnorstuvwxz/toutiao-multilevel-textclassfication-dataset. The Chinese Weibo dataset is addressed as: http://tcci.ccf.org.cn/conference/2014/pages/page04_sam.html. The Amazon review dataset is addressed as: http://jmcauley.ucsd. edu/data/amazon/.

\section{AUTHOR CONTRIBUTIONS}

SZ conceived and developed the theoretical framework of the manuscript, carried out experiment and data process, and drafted the manuscript.

Ahmad, M., Aftab, S., and Ali, I. (2017). Sentiment analysis of tweets using SVM. Int. J. Comput. Appl. 177, 975-8887. doi: 10.5120/ijca2017915758

Ahuja, R., Chug, A., Kohli, S., Gupta, S., and Ahuja, P. (2019). The impact of features extraction on the sentiment analysis. Procedia Comput. Sci. 152, 341-348. doi: 10.1016/j.procs.2019.05.008 
Almunirawi, K. M., and Maghari, A. (2016). A comparative study on serial decision tree classification algorithms in text mining. Int. J. Intell. Comput. Res. 7, 754-760. doi: 10.20533/ijicr.2042.4655.2016.0093

Alshamsi, A., Bayari, R., and Salloum, S. (2020). Sentiment analysis in English texts. Adv. Sci. Technol. Eng. Syst. J. 5, 1683-1689. doi: 10.25046/aj0506200

Bai, H., Yu, H., Yu, G., and Huang, X. (2020). A novel emergency situation awareness machine learning approach to assess flood disaster risk based on Chinese Weibo. Neural Comput. Appl. doi: 10.1007/s00521-020-05487-1 [Epub ahead of print].

Birjali, M., Kasri, M., and Beni-Hssane, A. (2021). A comprehensive survey on sentiment analysis: approaches, challenges and trends. Knowl. Based Syst. 226:107134. doi: 10.1016/j.knosys.2021.107134

Bozkurt, F., Çoban, O., Gunay, F., and Yucel Altay, S. (2019). High performance twitter sentiment analysis using CUDA based distance kernel on GPUs. Tehnicki Vjesnik-Technical Gazette 26, 1218-1227.

Chaturvedi, I., Satapathy, R., Cavallari, S., and Cambria, E. (2019). Fuzzy commonsense reasoning for multimodal sentiment analysis. Pattern Recognit. Lett. 125, 264-270. doi: 10.1016/j.patrec.2019.04.024

Chen, D., Xiong, Y., Yan, J., Xue, G. R., Wang, G., and Zheng, C. (2010). Knowledge transfer for cross domain learning to rank. Inf. Retrieval 13, 236-253.

Chen, L. C., Lee, C. M., and Chen, M. Y. (2020). Exploration of social media for sentiment analysis using deep learning. Soft Comput. 24, 8187-8197. doi: 10.1007/s00500-019-04402-8

Du, Y., He, M., Wang, L., and Zhang, H. (2020). Wasserstein based transfer network for cross-domain sentiment classification. Knowl. Based Syst. 204:106162. doi: 10.1016/j.knosys.2020.106162

Fei, R., Yao, Q., Zhu, Y., Xu, Q., and Hu, B. (2020). Deep learning structure for cross-domain sentiment classification based on improved cross entropy and weight. Sci. Program. 2020:3810261. doi: 10.1155/2020/3810261

Fu, Y., and Liu, Y. (2021). Cross-domain sentiment classification based on key pivot and non-pivot extraction. Knowl. Based Syst. 228:107280.

Gu, X., Fan, Y., Zhou, J., and Zhu, J. (2021). Optimized projection and fisher discriminative dictionary learning for EEG emotion recognition. Front. Psychol. 12:705528. doi: 10.3389/fpsyg.2021.705528

Gu, X., Lu, L., Qiu, S., Zou, Q., and Yang, Z. (2020). Sentiment key frame extraction in user-generated micro-videos via low-rank and sparse representation. Neurocomputing 410, 441-453. doi: 10.1016/j.neucom.2020.05.026

Gui, L., Lu, Q., Xu, R., Wei, Q., and Cao, Y. (2015). “Improving transfer learning in cross lingual opinion analysis through negative transfer detection," in International Conference on Knowledge Science, Engineering and Management, (China: Springer). doi: 10.1007/978-3-319-25159-2_36

Jia, X., Jin, Y., Li, N., and Su, X. (2018). Words alignment based on association rules for cross-domain sentiment classification. Front. Inf. Technol. Electronic.Eng. 19:260-272. doi: 10.1631/FITEE.1601679

Jiang, Y., Gu, X., Ji, D., and Jian, P. (2020). Smart diagnosis: a multiplesource transfer TSK fuzzy system for EEG seizure identification. ACM Trans. Multimed. Comput. Commun. Appl. 59, 1-21. doi: 10.1145/3340240

Jiang, Y., Gu, X., Wu, D., Hang, W., Xue, J., Qiu, S., et al. (2021). A novel negativetransfer-resistant fuzzy clustering model with a shared cross-domain transfer latent space and its application to brain CT image segmentation. IEEE/ACM Trans. Comput. Biol. Bioinform. 18, 40-52. doi: 10.1109/TCBB.2019.2963873

Kleinman, D. L., and Rao, P. K. (2003). Extensions to the Bartels-Stewart algorithm for linear matrix equations. IEEE Trans. Autom. Control 23, 85-87. doi: 10. 1109/TAC.1978.1101681

Lauren, P., Qu, G., Yang, J., Watta, P., Huang, G. B., and Lendasse, A. (2018). Generating word embeddings from an extreme learning machine for sentiment analysis and sequence labeling tasks. Cognit. Comput. 10, 625-638. doi: 10.1007/ s12559-018-9548-y

Liu, B., Xiao, Y., and Hao, Z. (2018). A selective multiple instance transfer learning method for text categorization problems. Knowl. Based Syst. 141, 178-187. doi: 10.1016/j.knosys.2017.11.019

Long, M., Wang, J., Ding, G., Pan, S. J., and Yu, P. S. (2014). Adaptation regularization: a general framework for transfer learning. IEEE Trans. Knowl. Data Eng. 26, 1076-1089. doi: 10.1109/TKDE.2013.111

López, M., Valdivia, A., Martínez-Cámara, E., Luzón, M. V., and Herrera, F. (2019). E2sam: evolutionary ensemble of sentiment analysis methods for domain adaptation. Inf. Sci. 480, 273-286. doi: 10.1016/j.ins.2018.12.038

Meng, J., Long, Y., Yu, Y., Zhao, D., and Liu, S. (2019). Cross-domain text sentiment analysis based on CNN_FT method. Information 10:162.
Ni, T., Ni, Y., Xue, J., and Wang, S. (2021). A domain adaptation sparse representation classifier for cross-domain electroencephalogram-based emotion classification. Front. Psychol. 12:721266. doi: 10.3389/fpsyg.2021. 721266

Pan, S. J., Ni, X., Sun, J. T., Yang, Q., and Chen, Z. (2010). "Cross-domain sentiment classification via spectral feature alignment," in Proceedings of the 19th International Conference on World Wide Web, (Carolina: ACM Digital Library), 26-30. doi: 10.1145/1772690.1772767

Shekhar, S., Patel, V. M., Nguyen, H. V., and Chellappa, R. (2013). "Generalized domain-adaptive dictionaries," in IEEE Conference on Computer Vision and Pattern Recognition, (Portland: IEEE), 361-368. doi: 10.1109/CVPR.2013.53

Tang, H., Mi, Y., Xue, F., and Cao, Y. (2021). Graph Domain Adversarial Transfer Network for Cross-Domain Sentiment Classification. IEEE Access 9, 3305133060. doi: 10.1109/ACCESS.2021.3061139

Tian, F., Wu, F., Fei, X., Shah, N., and Wang, Y. (2019). Improving generalization ability of instance transfer-based imbalanced sentiment classification of turnlevel interactive Chinese texts. Serv. Oriented Comput. Appl. 13, 155-167. doi: 10.1007/s11761-019-00264-y

Unnikrishnan, P., Govindan, V. K., and Madhu Kumar, S. (2019). Enhanced sparse representation classifier for text classification. Expert Syst. Appl. 129, 260-272. doi: 10.1016/j.eswa.2019.04.003

Waheeb, S. A., Ahmed Khan, N., Chen, B., and Shang, X. (2020). Machine Learning Based Sentiment Text Classification for Evaluating Treatment Quality of Discharge Summary. Information 11:281. doi: 10.3390/infol1050281

Wang, L., Niu, J., Song, H., and Atiquzzaman, M. (2018). SentiRelated: a crossdomain sentiment classification algorithm for short texts through sentiment related index. J. Netw. Comput. Appl. 101, 111-119. doi: 10.1016/j.jnca.2017. 11.001

Wen, J., Zhang, G., Zhang, H., Yin, W., and Ma, J. (2020). Speculative text mining for document-level sentiment classification. Neurocomputing 412, 52-62. doi: 10.1016/j.neucom.2020.06.024

Wu, Q., and Tan, S. (2011). A two-stage framework for cross-domain sentiment classification. Expert Syst. Appl. 38, 14269-14275. doi: 10.1016/j.eswa.2011.04. 240

Xia, R., Zong, C., Hu, X., and Cambria, E. (2013). Feature ensemble plus sample selection: domain adaptation for sentiment classification. IEEE Intell. Syst. 28, 10-18. doi: 10.1109/MIS.2013.27

Zhang, H., Sun, S., Hu, Y., Liu, J., and Guo, Y. (2020). Sentiment classification for Chinese text based on interactive multitask learning. IEEE Access 8, 129626129635. doi: 10.1109/ACCESS.2020.3007889

Zhang, K., Hei, X., Fei, R., Guo, Y., and Jiao, R. (2021). "Cross-domain text classification based on BERT model," in International Conference on Database Systems for Advanced Applications, (Taipei: Springer), doi: 10.1007/978-3-03073216-5_14

Zhang, S., Wei, Z., Yin, W., and Tao, L. (2018). Sentiment analysis of Chinese micro-blog text based on extended sentiment dictionary. Future Gener. Comput. Syst. 81, 395-403. doi: 10.1016/j.future.2017.09.048

Zhao, C., Wang, S., Li, D., Liu, X., Yang, X., and Liu, J. (2021). Cross-domain sentiment classification via parameter transferring and attention sharing mechanism. Inf. Sci. 578, 281-296. doi: 10.1016/j.ins.2021.07.001

Zheng, X., Lin, L., Liu, B., Xiao, Y., and Xiong, X. (2019). A multi-task transfer learning method with dictionary learning. Knowl. Based Syst. 191:105233.

Conflict of Interest: The author declares that the research was conducted in the absence of any commercial or financial relationships that could be construed as a potential conflict of interest.

Publisher's Note: All claims expressed in this article are solely those of the authors and do not necessarily represent those of their affiliated organizations, or those of the publisher, the editors and the reviewers. Any product that may be evaluated in this article, or claim that may be made by its manufacturer, is not guaranteed or endorsed by the publisher.

Copyright (C) 2021 Zhang. This is an open-access article distributed under the terms of the Creative Commons Attribution License (CC BY). The use, distribution or reproduction in other forums is permitted, provided the original author(s) and the copyright owner(s) are credited and that the original publication in this journal is cited, in accordance with accepted academic practice. No use, distribution or reproduction is permitted which does not comply with these terms. 